\title{
Proposal for new selection criteria considering pre-transplant muscularity and visceral adiposity in living donor liver transplantation
}

\section{AUTHOR(S):}

Hamaguchi, Yuhei; Kaido, Toshimi; Okumura, Shinya;

Kobayashi, Atsushi; Shirai, Hisaya; Yao, Siyuan; Yagi,

Shintaro; Kamo, Naoko; Okajima, Hideaki; Uemoto, Shinji

\section{CITATION:}

Hamaguchi, Yuhei ...[et al]. Proposal for new selection criteria considering pre-transplant muscularity and visceral adiposity in living donor liver transplantation. Journal of Cachexia, Sarcopenia and Muscle 2018, 9(2): 246-254

\section{ISSUE DATE:}

2018-04

URL:

http://hdl.handle.net/2433/230959

\section{RIGHT:}

(C) 2018 The Authors. Journal of Cachexia, Sarcopenia and Muscle published by John Wiley \& Sons Ltd on behalf of the Society on Sarcopenia, Cachexia and Wasting Disorders. This is an open access article under the terms of the Creative Commons Attribution - NonCommercial - NoDerivs License, which permits use and distribution in any medium, provided the original work is properly cited, the use is non - commercial and no modifications or adaptations are made. 


\title{
Proposal for new selection criteria considering pre-transplant muscularity and visceral adiposity in living donor liver transplantation
}

\author{
Yuhei Hamaguchi, Toshimi Kaido*, Shinya Okumura, Atsushi Kobayashi, Hisaya Shirai, Siyuan Yao, Shintaro Yagi iD, \\ Naoko Kamo, Hideaki Okajima \& Shinji Uemoto
}

Division of Hepato-Biliary-Pancreatic Surgery and Transplantation, Department of Surgery, Graduate School of Medicine, Kyoto University, Kyoto, Japan

\begin{abstract}
Background The significance of pre-operative body composition has recently attracted much attention in various diseases. However, cut-off values for these parameters remain undetermined, and these factors are not currently included in selection criteria for recipients of living donor liver transplantation (LDLT).

Methods Using computed tomography of 657 donors for LDLT, skeletal muscle mass, muscle quality, and visceral adiposity were evaluated by using skeletal muscle mass index (SMI), intramuscular adipose tissue content (IMAC), and visceral-to-subcutaneous adipose tissue area ratio (VSR). Sex-specific cut-offs for SMI, IMAC, and VSR were determined, and correlations with outcomes after LDLT in 277 recipients were examined with the aim of establishing new selection criteria for LDLT. Results On the basis of younger donor data, we determined sex-specific cut-off values for low SMI, high IMAC, and high VSR (mean \pm 2 standard deviations). Patients with all three factors showed the lowest survival rate after LDLT (1 year survival rate, 41.2\%; $P<0.001)$. On multivariate analysis, low SMI $(P=0.002)$, high IMAC $(P=0.002)$, and high VSR $(P=0.001)$ were identified as independent risk factors for mortality after LDLT. Based on these findings, we have excluded patients showing all three factors (low SMI, high IMAC, and high VSR) as candidates for LDLT since October 2016.

Conclusions Using cut-off values determined from healthy donors, we have established new selection criteria for LDLT including body composition, which should improve post-transplant outcomes.

Keywords Cut-off values; Intramuscular adipose tissue content; Liver transplantation; Sarcopenia; Skeletal muscle mass index; Visceral-to-subcutaneous adipose tissue area ratio
\end{abstract}

Received: 25 April 2017; Revised: 18 October 2017; Accepted: 20 November 2017

*Correspondence to: Toshimi Kaido, MD, PhD, Division of Hepato-Biliary-Pancreatic Surgery and Transplantation, Department of Surgery, Graduate School of Medicine, Kyoto University, 54 Kawahara-cho, Shogoin, Sakyo-ku, Kyoto 606-8507, Japan. Fax: +81-75-751-4263, Email: kaido@kuhp.kyoto-u.ac.jp

\section{Introduction}

Sarcopenia is characterized by age-related progressive and generalized decline in skeletal muscle mass and muscle strength and has been accepted worldwide as a new geriatric syndrome. ${ }^{1}$ Recent studies have reported significant associations between sarcopenia and poor outcomes in various diseases. $^{2-7}$ In the field of liver transplantation (LT), our recent studies have demonstrated that pre-operative low muscularity (low muscle mass and low muscle quality) shows a close correlation with post-transplant mortality. ${ }^{8,9}$ On the other hand, high visceral adiposity calculated as the visceral-to-subcutaneous adipose tissue area ratio (VSR) has been reported as a useful predictor of poor outcomes in several cancers. ${ }^{10-12}$ However, these body compositions have not been included in the current selection criteria for recipients for LT, because the method for measuring muscularity and visceral adiposity varies between investigations and because universally accepted cut-off values for these factors remain undetermined. 
The European Working Group on Sarcopenia in Older People has proposed that measuring and evaluating muscle mass, muscle strength, and physical performance are necessary in order to diagnose sarcopenia. ${ }^{1}$ Among various imaging modalities, such as dual-energy X-ray absorptiometry, computed tomography (CT), magnetic resonance imaging, and bioimpedance analysis, CT seems the best suited to measuring skeletal muscle mass in clinical settings, particularly in the surgical field, as CT is usually performed as part of the pre-operative work-up and during follow-up. Although several studies from Western countries have proposed different cut-offs for low skeletal muscle mass as calculated from $\mathrm{CT}_{1}, 3,5$ such values would differ from those in Asian populations, due to general differences in characteristics such as body size, lifestyles, and other factors. We have previously established new diagnostic criteria for low skeletal muscle mass by using the psoas muscle mass index from healthy young Asian adults, which was adopted in the Japan Society of Hepatology guidelines for sarcopenia in liver disease. ${ }^{13,14}$ However, it is usual for evaluating skeletal muscle mass to investigate all skeletal muscle areas at the third lumbar vertebra (L3) level, not only psoas muscle areas. $^{15}$ In addition, no studies have established cut-off values for evaluating muscle quality and visceral adiposity in a healthy general population.

The present study evaluated body composition including skeletal muscle mass, muscle quality, and visceral adiposity by using CT of healthy adult donors for living donor LT (LDLT), then established sex-specific cut-offs for these parameters. Furthermore, the impact of these parameters on outcomes after LDLT was investigated. On the basis of the results, we aimed to develop more objective selection criteria for recipients of LDLT considering pre-transplant nutritional and physical statuses.

\section{Patients and methods}

\section{Study subjects}

Between April 2005 and July 2016, a total of 675 adult (age $\geq 20$ years) donors were admitted to Kyoto University Hospital for adult or paediatric LDLT. Eighteen patients who did not undergo pre-operative plain CT were excluded from analysis. This retrospective study therefore analysed data from 657 donors (331 men, 326 women) to investigate muscularity and adiposity. The median age of the donors was 39 years [interquartile range (IQR), 31-52 years]. Numbers of donors in each age decade were as follows: 20-29 years, 126 (19.2\%); 30-99 years, 209 (31.8\%); 40-49 years, 133 (20.2\%); 50-59 years, 136 (20.7\%); and $\geq 60$ years, 53 (8.1\%).

To evaluate the validity of cut-offs for body composition parameters, we investigated data from patients who underwent adult-to-adult LDLT. A total of 301 adult patients underwent LDLT in our institution between January 2008 and July 2016. Of these, 24 patients who did not undergo pre-operative plain CT at the L3 level were excluded from analysis. Therefore, 277 patients were enrolled in this study. Median duration of follow-up for these recipients was 26.1 months (IQR, 7.8-64.1 months). Selection criteria for donors and recipients have been described previously. ${ }^{16,17}$ In brief, living donors must fulfil the following acceptance criteria: (i) a relationship with the recipient within the third civil degree and strong voluntary will to donate part of the liver and (ii) no medical disorder that would significantly increase the perioperative risk. The exclusion criterion for living liver donation was a planned remnant liver volume $<30 \%$ of whole liver volume as estimated from pre-operative CT volumetry. Exclusion criteria for recipients for LDLT were as follows: (i) acute heart and/or renal failure, (ii) ongoing active infection, (iii) extrahepatic malignancy, or (iv) hepatic malignancy with major vascular invasion. All study protocols were approved by the Ethics Committee of Kyoto University (approval \#R0061) and were conducted in accordance with the 1996 revision of the Declaration of Helsinki.

\section{Image analysis}

All CTs were performed by using a multidetector-row CT scanner (Aquilion 64; Toshiba Medical Systems, Tochigi, Japan). The technical parameters for CT were tube voltage, $120 \mathrm{kV}$; detector configuration, $0.5 \mathrm{~mm} \times 64$ rows; tube current modulation, $0.5 \mathrm{~s} /$ rotation (gantry rotation); and reconstruction thickness, $5 \mathrm{~mm}$.

Using cross-sectional CT at the L3 level, skeletal muscle and adipose tissue areas were examined by using AQUARIUS INTUITION software (TeraRecon, San Mateo, CA). The AQUARIUS INTUITION is an iNtuition client viewer with advanced visualization capabilities. With this software, areas of skeletal muscle, visceral, and subcutaneous adipose tissue can be easily and automatically quantified by using the CT attenuation values peculiar to these tissues. Skeletal muscle areas including psoas, erector spinae, quadratus lumborum, transversus abdominis, external and internal obliques, and rectus abdominis were identified and quantified by using attenuation values of -29 to 150 Hounsfield units (HU) (Figure 1 A). ${ }^{18}$ Skeletal muscle mass was evaluated as the skeletal muscle mass index (SMI) calculated by normalizing skeletal muscle areas to the square of the patient's height $\left(\mathrm{cm}^{2} /\right.$ $\mathrm{m}^{2}$ ). Muscle quality was examined as intramuscular adipose tissue content (IMAC) at the L3 level. As previously described, IMAC was calculated by dividing the CT attenuation value of the multifidus muscles by that of subcutaneous fat (Figure 1 B). ${ }^{9}$ A higher IMAC indicates a greater amount of adipose tissue within skeletal muscle and thus a lower quality of skeletal muscle (muscle steatosis). In addition, subcutaneous and 
Figure 1 Cross-sectional computed tomography at the level of the third lumbar vertebra. (A) Skeletal muscle areas were identified and quantified by using a computed tomography attenuation value of -29 to $150 \mathrm{HU}$. (B) Computed tomography attenuation values of subfascial muscular tissue in the multifidus muscle and subcutaneous fat (four small circles) were examined to calculate IMAC. (C) Subcutaneous adipose tissue areas were quantified by using attenuation values of -190 to $-30 \mathrm{HU}$. (D) Visceral adipose tissue areas were quantified by using attenuation values of -150 to $-50 \mathrm{HU}$. $\mathrm{HU}$, Hounsfield units; IMAC, intramuscular adipose tissue content.

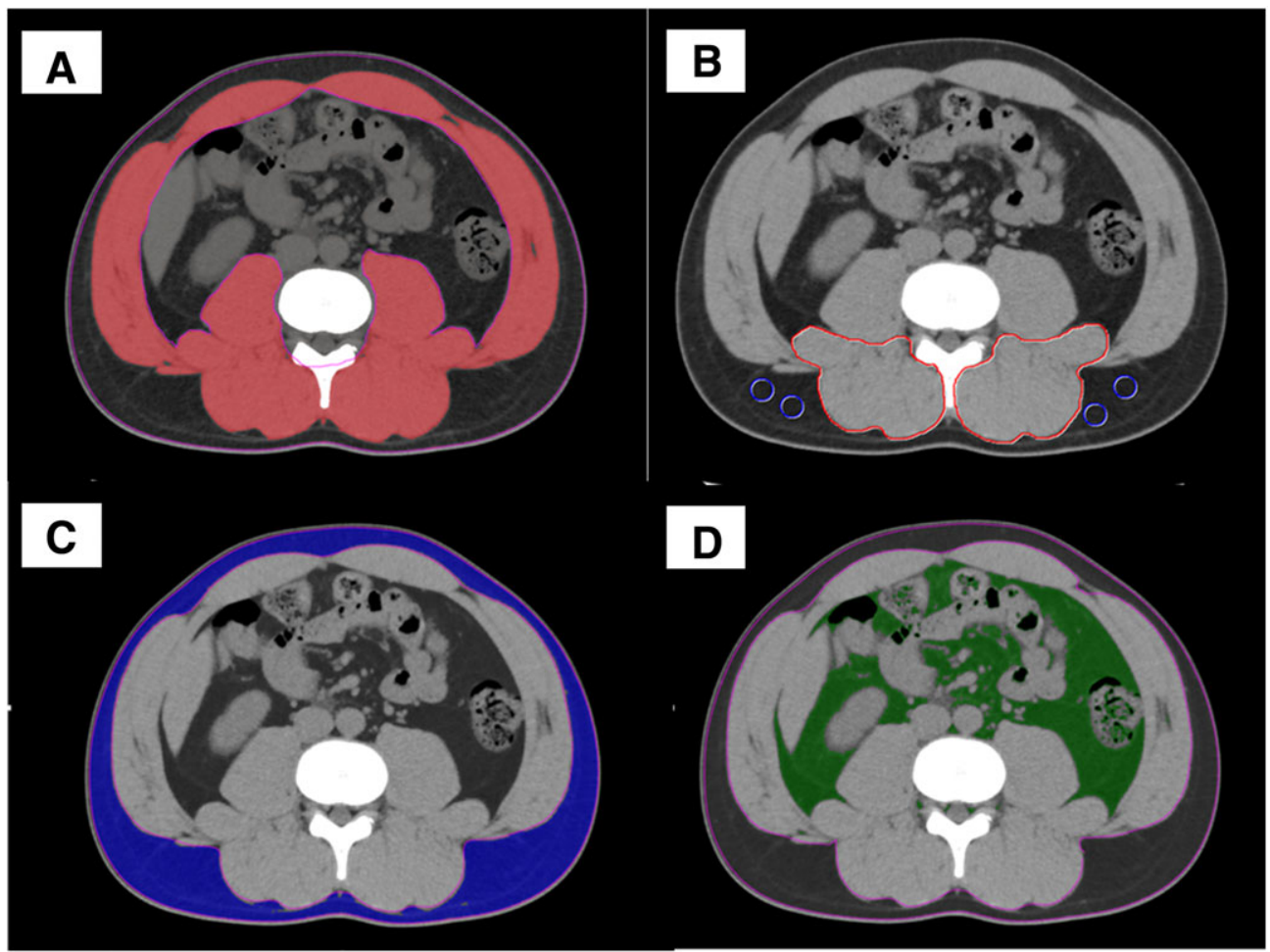

visceral adipose tissue areas were quantified by using attenuation values of -190 to $-30 \mathrm{HU}$ (Figure 1C) and -150 to $-50 \mathrm{HU}$ (Figure 1D), respectively. ${ }^{18,19}$ VSR, which indicates visceral adiposity, was calculated by dividing visceral adipose tissue area by subcutaneous adipose tissue area. ${ }^{10}$

\section{Analysed parameters}

First, distributions of SMI, IMAC, and VSR according to sex and donor age were evaluated by using the data from the 657 donors, then sex-specific cut-offs of these parameters were established. Second, the overall survival rate after LDLT was investigated in the 277 recipients classified according to SMI, IMAC, and VSR. Moreover, prognostic factors were analysed on the basis of the following variables: recipient age $(\geq 50$ years vs. $<50$ years), donor age $(\geq 50$ years vs. $<50$ years), sex (male vs. female), original disease, $A B O$ compatibility (identical/compatible vs. incompatible), Model for End-stage Liver Disease (MELD) score ( $\geq 20$ vs. $<20$ ), Child-Pugh classification (A/B vs. C), graft type (right vs. left), graft-to-recipient body weight ratio $(\geq 0.8 \%$ vs. $<0.8 \%)$, duration of surgery ( $\geq 12 \mathrm{~h}$ vs. $<12 \mathrm{~h}$ ), estimated blood loss $(\geq 10 \mathrm{~L}$ vs. $<10 \mathrm{~L}$ ), skeletal muscle mass (low SMI vs. normal
SMI), skeletal muscle quality (high IMAC vs. normal IMAC), and visceral adiposity (high VSR vs. normal VSR). Finally, on the basis of these results, we established new selection criteria for recipients of LDLT.

\section{Statistical analysis}

Continuous data are presented as median (IQR) and were non-parametrically analysed by using the Mann-Whitney $U$-test. Categorical variables were compared by using the $\chi^{2}$ test or Fisher's exact test, as appropriate. Correlations between continuous variables were assessed by using Pearson correlation coefficients. Cumulative overall survival rates were calculated by using Kaplan-Meier methods, with differences between curves evaluated by using the log-rank test. Any variable identified as significant $(P<0.05)$ or showing values of $P<0.10$ on univariate analyses was considered a candidate for multivariate Cox regression analysis, and the results are shown as hazard ratios (HRs) with $95 \%$ confidence intervals. A value of $P<0.05$ was considered significant. All statistical data were generated by using JMP PRO version 12 software (SAS Institute, Cary, NC) and PRISM 6 software (GraphPad Software, La Jolla, CA). 


\section{Results}

\section{Distributions of body composition according to sex and donor age}

Skeletal muscle mass index, IMAC, and VSR differed significantly between men and women ( $P<0.001$ each) (Table 1$)$. In addition, in both men and women, weak-to-moderate, but statistically highly significant relationships were observed between donor age and SMI (men: $r=-0.291, P<0.001$, Figure 2A; women: $r=-0.254, P<0.001$, Figure 2B), IMAC (men: $r=0.533, P<0.001$, Figure 3A; women: $r=0.492, P<0.001$, Figure 3B), and VSR (men: $r=0.618, P<0.001$, Figure 4A; women: $r=0.526, P<0.001$, Figure 4B). SMI, IMAC, and VSR in younger donors ( $<50$ years) thus differed significantly from those in older donors ( $\geq 50$ years) in both men $(P<0.001$ each; Table 2$)$ and women $(P<0.001$ each; Table 2$)$.

Sex-specific cut-offs for skeletal muscle mass index, intramuscular adipose tissue content, and visceral-to-subcutaneous adipose tissue area ratio

Based on these results, the sex-specific cut-off values of SMI for low skeletal muscle mass were defined as more than two standard deviations (SDs) below the mean SMI of younger donors $(<50$ years), resulting in cut-offs of $40.31 \mathrm{~cm}^{2} / \mathrm{m}^{2}$ for men and $30.88 \mathrm{~cm}^{2} / \mathrm{m}^{2}$ for women. Similarly, high IMAC was defined as more than the 2 SDs above the mean, resulting in cut-offs of -0.358 for men and -0.229 for women, while cut-offs for VSR were 1.325 for men and 0.710 for women.

\section{Impact of body composition on outcomes after living donor liver transplantation}

Using the calculated cut-off values for body composition parameters, we investigated the impact of pre-transplant muscularity and visceral adiposity on outcomes after LDLT. Among the 277 LDLT recipients, 55 (20.0\%), 121 (43.7\%), and 83 (30.0\%) patients exhibited low SMI, high IMAC, and high VSR, respectively. The overall survival rate was significantly lower for each group of patients with low SMI $(P<0.001)$, high IMAC $(P<0.001)$, or high VSR $(P<0.001)$ compared with the respective normal groups. In addition, low SMI, high IMAC, and high VSR contributed to an increased risk of post-LDLT mortality in an additive manner (Figure 5). Patients beyond all three cut-offs ( $n=17,6.1 \%$ ) showed the lowest survival rate after LDLT (1 year survival, $41.2 \% ; P<0.001$ ). A total of 70 patients died in this follow-up period. The causes of death for 70

Table 1 Body composition classified according to sex

\begin{tabular}{lccc}
\hline Body composition & Male & Female & $P$ value \\
\hline $\begin{array}{l}\text { Pre-transplant SMI } \\
\left(\mathrm{cm}^{2} / \mathrm{m}^{2}\right) \text { median (IQR) }\end{array}$ & $52.37(48.17$ to 56.77$)$ & $39.41(36.29$ to 42.77$)$ & $<0.001$ \\
$\begin{array}{l}\text { Pre-transplant IMAC } \\
\text { median (IQR) }\end{array}$ & $-0.452(-0.512$ to -0.401$)$ & $-0.339(-0.412$ to -0.281$)$ & $<0.001$ \\
$\begin{array}{l}\text { Pre-transplant VSR } \\
\text { median (IQR) }\end{array}$ & $0.719(0.502$ to 1.053$)$ & $0.372(0.268$ to 0.560$)$ & $<0.001$
\end{tabular}

IQR, interquartile range; SMI, skeletal muscle mass index; IMAC, intramuscular adipose tissue content; VSR, visceral-to-subcutaneous adipose tissue area ratio.

Figure 2 Correlations between SMI and donor age classified according to sex. Significant negative relationships are observed between SMI and donor age in both (A) men and (B) women. SMI, skeletal muscle mass index.

A

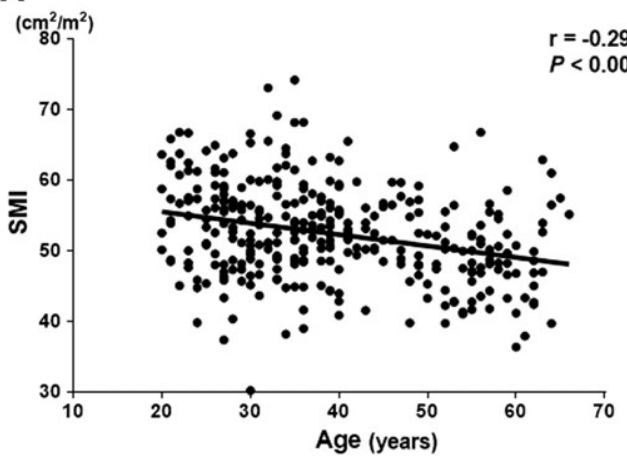

B

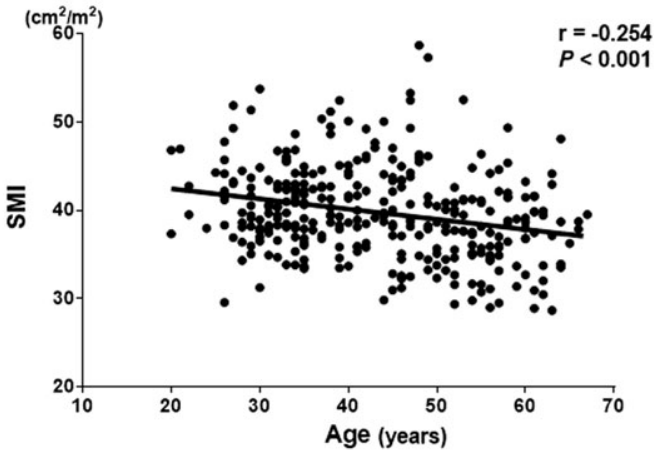


Figure 3 Correlations between IMAC and donor age classified according to sex. Significant positive relationships are observed between IMAC and donor age in both (A) men (B) women. IMAC, intramuscular adipose tissue content.
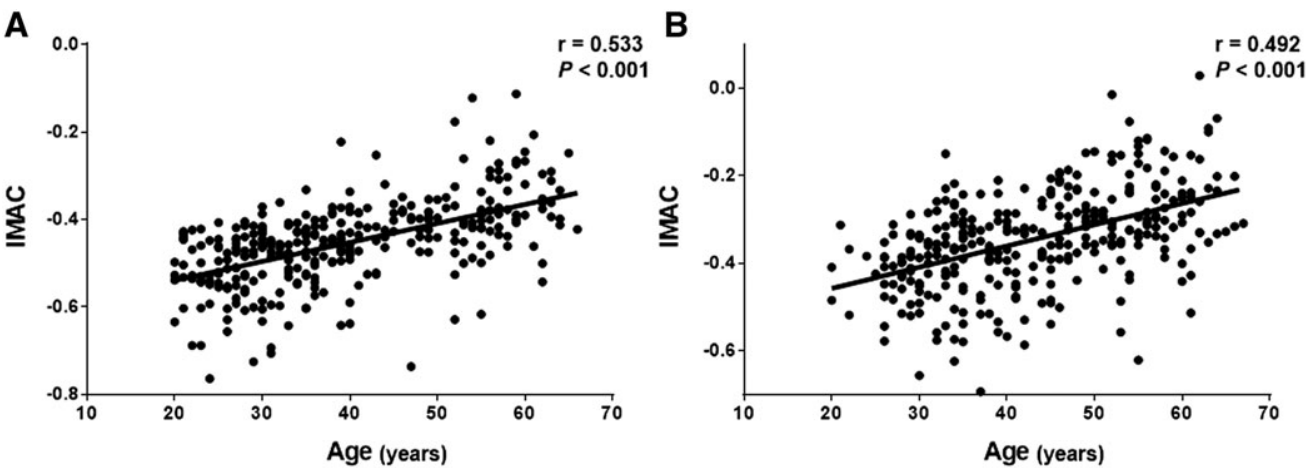

Figure 4 Correlations between VSR and donor age classified according to sex. Significant positive relationships are observed between VSR and donor age in both (A) men and (B) women.VSR, visceral-to-subcutaneous adipose tissue area ratio.

A

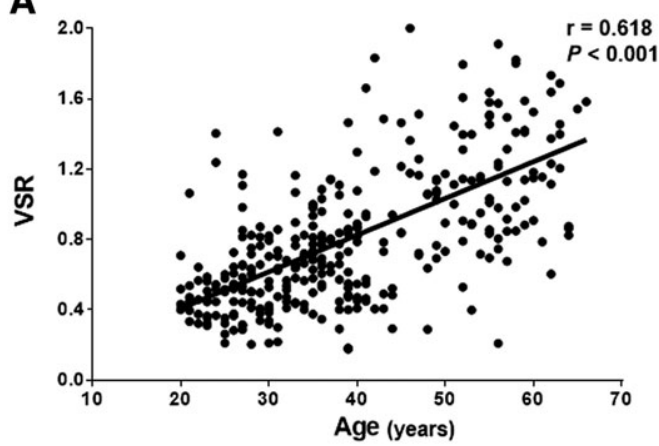

B

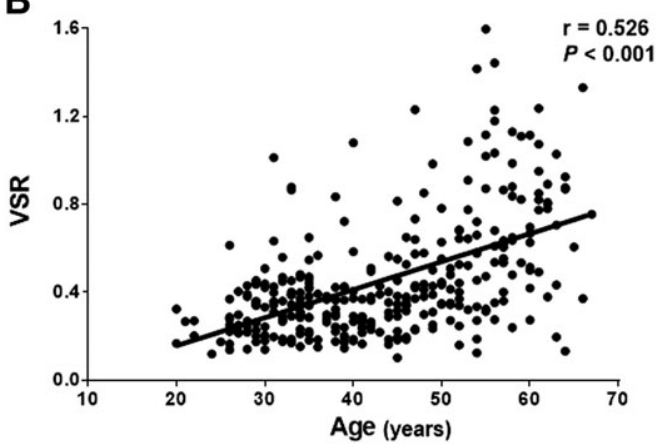

Table 2 Body composition compared between younger ( $<50$ years) and older ( $\geq 50$ years) donors

\begin{tabular}{|c|c|c|c|}
\hline \multicolumn{4}{|l|}{ Male } \\
\hline Body composition & Younger & Older & $P$ value \\
\hline $\begin{array}{l}\text { Pre-transplant SMI }\left(\mathrm{cm}^{2} / \mathrm{m}^{2}\right) \text { median (IQR) } \\
\text { Pre-transplant IMAC median (IQR) } \\
\text { Pre-transplant VSR median (IQR) }\end{array}$ & $\begin{array}{l}53.38(48.91 \text { to } 57.43) \\
-0.465(-0.529 \text { to }-0.426) \\
0.623(0.448 \text { to } 0.825)\end{array}$ & $\begin{array}{l}48.33(44.39 \text { to } 52.39) \\
-0.389(-0.430 \text { to }-0.313) \\
1.147(0.883 \text { to } 1.454)\end{array}$ & $\begin{array}{l}<0.001 \\
<0.001 \\
<0.001\end{array}$ \\
\hline \multicolumn{4}{|l|}{ Female } \\
\hline Body composition & Younger & Older & $P$ value \\
\hline $\begin{array}{l}\text { Pre-transplant SMI }\left(\mathrm{cm}^{2} / \mathrm{m}^{2}\right) \text { median (IQR) } \\
\text { Pre-transplant IMAC median (IQR) } \\
\text { Pre-transplant VSR median (IQR) }\end{array}$ & $\begin{array}{l}40.64(37.89 \text { to } 44.03) \\
-0.375(-0.447 \text { to }-0.313) \\
0.323(0.232 \text { to } 0.421)\end{array}$ & $\begin{array}{l}37.49(34.39 \text { to } 40.30) \\
-0.292(-0.336 \text { to }-0.222) \\
0.610(0.401 \text { to } 0.858)\end{array}$ & $\begin{array}{l}<0.001 \\
<0.001 \\
<0.001\end{array}$ \\
\hline
\end{tabular}

ipose tissue area ratio.

patients were sepsis $(n=23)$, pulmonary complications ( $n=13$ ), graft failure including antibody-mediated rejection and chronic rejection $(n=17)$, cerebral bleeding $(n=8)$, hepatocellular carcinoma recurrence $(n=2)$, and others $(n=7)$. As the number of risk factors increased, significantly more patients died from infectious complications, such as sepsis, biliary infection, and pneumonia (none: $1.0 \%$, one: 10.2\%, two: $32.0 \%$, three: $47.1 \% ; P<0.001$ ). Major posttransplant complication rates (including grades III and IV of the Dindo and Clavien classification) and post-transplant hospital stay were similar among four groups $(P=0.262$, $P=0.197$, respectively). 
Figure 5 Overall survival rates in patients classified by number of body composition variables. Overall survival rates after LDLT decreased significantly with an increasing number of prognostic body composition factors (low SMI, high IMAC, and high VSR) $(P<0.001)$. IMAC, intramuscular adipose tissue content; LDLT, living donor liver transplantation; SMI, skeletal muscle mass index; VSR, visceral-to-subcutaneous adipose tissue area ratio.

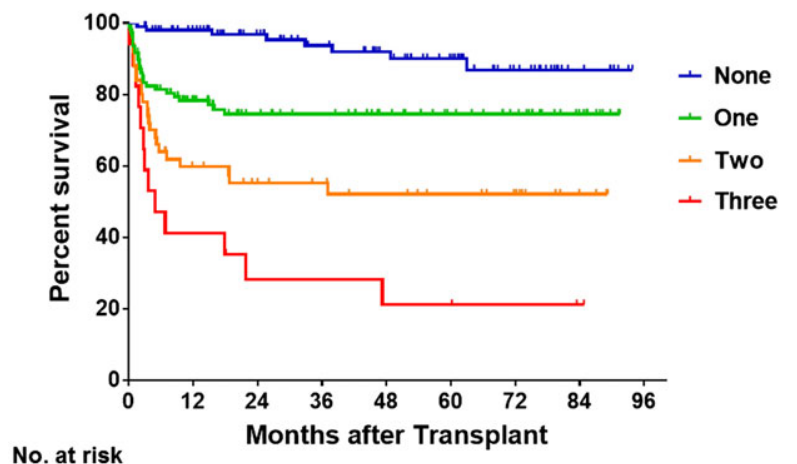

\begin{tabular}{lcrrr} 
None & 102 & 70 & 47 & 22 \\
One & 108 & 52 & 37 & 23 \\
Two & 50 & 22 & 17 & 11 \\
Three & 17 & 5 & 4 & 3 \\
\hline
\end{tabular}

\section{Risk factors for poor survival after living donor liver transplantation}

Univariate Cox regression analysis identified that left lobe graft $(P=0.030)$, pre-operative low SMI $(P<0.001)$, pre-operative high IMAC $(P<0.001)$, and pre-operative high VSR $(P<0.001)$ were all significant risk factors for post-LDLT mortality (Table 3). Multivariate analysis identified pre-operative low SMI (HR = 2.355, $P=0.002$ ), high IMAC $(\mathrm{HR}=2.179, P=0.002)$, and high VSR $(\mathrm{HR}=2.373$, $P=0.001$ ) as poor prognostic factors after LDLT (Table 3 ).

Based on these results, we have excluded patients beyond all three cut-offs (low SMI, high IMAC, and high VSR) as candidates for LDLT since October 2016.

\section{Discussion}

Liver transplantation is currently the only curative treatment providing a chance of long-term survival among patients with end-stage liver disease, advanced hepatocellular carcinoma, or acute liver failure. ${ }^{20}$ Advances in surgical techniques, perioperative management, organ preservation, and immunosuppression have dramatically improved patient survival after LT in the last few decades, and the number of LT candidates is now steadily growing. ${ }^{21,22}$ However, despite such advances, some patients still exhibit poor prognosis after LT, and we considered that some risk factors for poor outcomes were not reflected by the current selection criteria for LT. The MELD scoring system is currently the most widely accepted method for predicting mortality among patients awaiting LT. ${ }^{23}$ Although MELD score is calculated by using the three objective parameters of international normalized ratio of prothrombin time, serum bilirubin, and serum creatinine, the predictive ability of MELD score for post-LT outcomes remains controversial. ${ }^{21,24-}$ ${ }^{27}$ Proper extraction of risk factors for death after LT is thus urgently needed to enable the establishment of objective and universal selection criteria. Based on our previous study, ${ }^{8}$ we added a new indication of 'patients who can walk unaided' to our selection criteria for LT in 2013, resulting in better outcomes after LDLT. Although this new indication has significantly improved post-transplant outcomes in our institution, more objective and universal selection criteria that include sarcopenic factors should be established.

Several Japanese studies have proposed cut-offs to define low skeletal muscle mass by using CT. ${ }^{14,28}$ However, Nishikawa et al. evaluated skeletal muscle mass in patients with liver cirrhosis, not in the general population, ${ }^{14}$ while the other study used a small cohort of 45 healthy adults to investigate the relationship between skeletal muscle area and body surface. ${ }^{28}$ In addition, no studies have investigated muscle steatosis and visceral adiposity to establish cut-offs by using data from a general healthy population. Body compositions differ significantly between men and women. The present study also showed that SMI and VSR were significantly higher in men, and IMAC was significantly lower in men compared with that in women ( $P<0.001$ each). In addition, a continuous worsening of muscularity and visceral adiposity is generally seen with the ageing process. This change in body compositions has been shown to become pronounced around 50 years old, with faster progression noted after 60 years old. ${ }^{29,30}$ In the present study, all body compositions (SMI, IMAC, and VSR) in younger donors $(<50$ years) were significantly better than those in older donors ( $\geq 50$ years) in both men $(P<0.001$ each) and women $(P<0.001$ each). On the basis of these findings, we took into consideration these two key factors of sex and age to establish optimal cut-offs for the three body compositions. To the best of our knowledge, the present study is the first to evaluate muscularity and visceral adiposity by using CT in a large number of healthy Asian adults and to establish cut-offs for these parameters to define the low muscularity and high visceral adiposity.

Similar to our previous study, ${ }^{8,9}$ when using the new cutoffs, patients with low muscle mass and high muscle steatosis exhibited lower survival rates than the respective normal groups ( $P<0.001$ each). In addition, the overall survival rate was significantly lower in patients with high visceral adiposity than in those with normal VSR $(P<0.001)$. SMI, IMAC, and VSR were independent of each other, and no significant relationships were seen among these three factors (data not shown). Therefore, based on multivariate analysis investigating risk factors for post-LDLT mortality, we considered that incorporation of all three factors into new selection criteria for LT recipients was appropriate. The survival rate after LDLT 
Table 3 Univariate and multivariate analyses of prognostic factors for post-transplant survival

\begin{tabular}{|c|c|c|c|c|c|c|}
\hline \multirow[t]{2}{*}{ Variable } & \multicolumn{3}{|c|}{ Univariate } & \multicolumn{3}{|c|}{ Multivariate } \\
\hline & $\mathrm{HR}$ & $95 \% \mathrm{Cl}$ & $P$ Value & $\mathrm{HR}$ & $95 \% \mathrm{Cl}$ & $P$ Value \\
\hline \multicolumn{7}{|l|}{ Recipient age (years) } \\
\hline$<50(\mathrm{n}=97)$ & 1.000 & (referent) & & & & \\
\hline$\geq 50(n=180)$ & 0.699 & $0.436-1.136$ & 0.146 & & & \\
\hline \multicolumn{7}{|l|}{ Donor age (years) } \\
\hline$<50(n=162)$ & 1.000 & (referent) & & & & \\
\hline$\geq 50(n=115)$ & 1.134 & $0.703-1.815$ & 0.601 & & & \\
\hline \multicolumn{7}{|l|}{ Sex } \\
\hline Male $(n=134)$ & 1.000 & (referent) & & & & \\
\hline Female $(n=143)$ & 1.136 & $0.711-1.828$ & 0.594 & & & \\
\hline \multicolumn{7}{|l|}{ Original disease } \\
\hline $\mathrm{HCC}(\mathrm{n}=90)$ & 1.000 & (referent) & & & & \\
\hline HBV or HCV-associated LC $(n=50)$ & 1.050 & $0.527-2.016$ & 0.886 & & & \\
\hline PBC or PSC $(n=49)$ & 1.445 & $0.750-2.717$ & 0.265 & & & \\
\hline Others $(n=88)$ & 0.751 & $0.395-1.400$ & 0.369 & & & \\
\hline \multicolumn{7}{|l|}{ Period of LDLT } \\
\hline $2008-2010(n=130)$ & 1.000 & (referent) & & & & \\
\hline $2011-2015(n=147)$ & 0.842 & $0.519-1.360$ & 0.481 & & & \\
\hline \multicolumn{7}{|l|}{ ABO compatibility } \\
\hline Identical/compatible $(n=199)$ & 1.000 & (referent) & & 1.000 & (referent) & \\
\hline Incompatible $(n=78)$ & 1.616 & $0.982-2.605$ & 0.059 & 1.651 & $0.995-2.687$ & 0.052 \\
\hline \multicolumn{7}{|l|}{ MELD score } \\
\hline$<20(\mathrm{n}=179)$ & 1.000 & (referent) & & 1.000 & (referent) & \\
\hline$\geq 20(n=98)$ & 1.564 & $0.970-2.502$ & 0.067 & 1.347 & $0.822-2.189$ & 0.235 \\
\hline \multicolumn{7}{|l|}{ Child-Pugh classification } \\
\hline$A, B(n=99)$ & 1.000 & (referent) & & & & \\
\hline$C(n=178)$ & 1.025 & $0.634-1.695$ & 0.920 & & & \\
\hline \multicolumn{7}{|l|}{ GRWR (\%) } \\
\hline$<0.8(n=87)$ & 1.000 & (referent) & & & & \\
\hline$\geq 0.8(n=190)$ & 0.926 & $0.544-1.522$ & 0.768 & & & \\
\hline \multicolumn{7}{|l|}{ Graft type } \\
\hline Right $(n=157)$ & 1.000 & (referent) & & 1.000 & (referent) & \\
\hline Left $(n=120)$ & 1.684 & $1.053-2.709$ & 0.030 & 1.103 & $0.669-1.824$ & 0.701 \\
\hline \multicolumn{7}{|l|}{ Splenectomy } \\
\hline Yes $(n=171)$ & 1.000 & (referent) & & & & \\
\hline No $(n=106)$ & 1.115 & $0.680-1.797$ & 0.661 & & & \\
\hline \multicolumn{7}{|l|}{ PVP $(\mathrm{mmHg})$} \\
\hline$<15(n=223)$ & 1.000 & (referent) & & & & \\
\hline$\geq 15(n=54)$ & 1.576 & $0.897-2.642$ & 0.110 & & & \\
\hline \multicolumn{7}{|l|}{ Operative time (hour) } \\
\hline$<12(n=53)$ & 1.000 & (referent) & & & & \\
\hline$\geq 12(n=224)$ & 0.683 & $0.408-1.201$ & 0.178 & & & \\
\hline \multicolumn{7}{|l|}{ Operative blood loss (L) } \\
\hline$<10(n=193)$ & 1.000 & (referent) & & & & \\
\hline$\geq 10(n=84)$ & 0.820 & $0.473-1.365$ & 0.455 & & & \\
\hline \multicolumn{7}{|l|}{ Preoperative SMI } \\
\hline Normal $(n=222)$ & 1.000 & (referent) & & 1.000 & (referent) & \\
\hline $\operatorname{Low}(\mathrm{n}=55)$ & 3.084 & $1.883-4.964$ & $<0.001$ & 2.355 & $1.399-3.907$ & 0.002 \\
\hline Preoperative IMAC & & & & & & \\
\hline Normal $(n=156)$ & 1.000 & (referent) & & 1.000 & (referent) & \\
\hline $\operatorname{High}(n=121)$ & 2.641 & $1.633-4.369$ & $<0.001$ & 2.179 & $1.336-3.632$ & 0.002 \\
\hline Preoperative VSR & & & & & & \\
\hline Normal $(n=194)$ & 1.000 & (referent) & & 1.000 & (referent) & \\
\hline High $(n=83)$ & 3.496 & $2.185-5.643$ & $<0.001$ & 2.373 & $1.441-3.939$ & 0.001 \\
\hline
\end{tabular}

$\mathrm{Cl}$, confidence interval; GRWR, graft-to-recipient body weight ratio; HBV, hepatitis B virus; HCC, hepatocellular carcinoma; HCV, hepatitis $C$ virus; HR, hazard ratio; IMAC, intramuscular adipose tissue content; LC, liver cirrhosis; LDLT, living donor liver transplantation; MELD, Model for End-stage Liver Disease; PBC, primary biliary cirrhosis; PSC, primary sclerosing cholangitis; PVP, portal venous pressure; SMI, skeletal muscle mass index; VSR, visceral-to-subcutaneous adipose tissue area ratio.

gradually declined with increases in the number of risk factors (low SMI, high IMAC, and high VSR) (Figure 5). One-year survival rates among patients beyond none, one, two, or three of these cut-offs were $98.0 \%, 78.3 \%, 59.7 \%$, and $41.2 \%$, respectively. Based on these results, although the number of patients meeting all three factors was small
( $n=17,6.1 \%$ ), we considered that these patients, showing a 1 year survival rate below $50 \%$, should be excluded as candidates for LDLT. In October 2016, we added this objective indication to our selection criteria for recipients for LDLT, excluding patients meeting all three of low SMI, high IMAC, and high VSR. However, these parameters might be able to 
be improved with the aid of pre-transplant nutritional intervention and rehabilitation, and outcomes in such cases have yet to be fully investigated. Further prospective evaluations for the validity of new criteria are needed.

The mechanisms by which low muscularity and visceral adiposity adversely affect post-transplant mortality are not yet fully understood. Skeletal muscle loss with increasing adipose tissue in muscles results in the synthesis and secretion of pro-inflammatory adipokines and declines in myokine concentrations. ${ }^{31}$ This imbalance between adipokines and myokines in older adults or sarcopenic populations has been shown to lead to immunosenescence, particularly of the natural killer lymphocytes involved in innate immunity. ${ }^{32}$ On the other hand, visceral adiposity paradoxically impairs immune function by altering leukocyte counts, as well as cell-mediated immune responses, although excessive adipose tissue activates various kinds of immune cells through increases in leptin and decreases in adiponectin. ${ }^{33}$ On the basis of these findings, we speculate that low muscularity and visceral adiposity induce an inflammatory microenvironment through imbalances between adipokines and other cytokines, which could impair immune function and increase mortality risk.

The findings of this study should be considered in light of several limitations. It is necessary to consider whether the use of 2 SDs beyond the mean of younger donors was adequate in setting cut-offs for SMI, IMAC, and VSR. In our previous studies, ${ }^{9,34-36}$ cut-offs for body composition were based on receiver operating characteristic curves. Although the use of receiver operating characteristic curves provides a more accurate and objective method than the use of SDs for designing cut-offs, these values differ markedly between study populations. Therefore, to avoid this potential problem in using data from healthy adults, the present study aimed to establish optimal cut-offs that could be universally applied to other research into body composition. A previous study defined sex-specific cut-offs for low skeletal muscle mass as more than 2 SDs below the means of young adults. ${ }^{37}$ In addition, the Asian Working Group for Sarcopenia has also recommended using 2 SDs below the mean muscle mass of a young reference group or the lower quintile when determining cut-offs. ${ }^{38}$ In the present study, SMI, IMAC, and VSR differed significantly between younger ( $<50$ years) and older ( $\geq 50$ years) donors. We therefore decided to define donors $<50$ years old as the reference group and to use these values to determine cut-offs.

In conclusion, the present study is the first to evaluate muscularity and visceral adiposity by using CT in a large number of healthy Japanese individuals and to establish optimal cut-offs for these parameters to define low muscularity and high visceral adiposity. Furthermore, using these cut-offs, we investigated risk factors for post-LDLT mortality and extracted the group that exhibited the poorest prognosis after LT, leading to the establishment of our new selection criteria for recipients of LDLT.

\section{Acknowledgements}

The authors of this paper certify that they comply with the ethical guidelines for authorship and publishing of the Journal of Cachexia, Sarcopenia and Muscle. ${ }^{39}$ The authors would like to thank Ms Mayumi Kawashima for her help with collecting the data for this study.

\section{Conflict of interest}

The authors declare no conflicts of interest.

\section{References}

1. Cruz-Jentoft AJ, Baeyens JP, Bauer JM Boirie Y, Cederholm T, Landi F, et al. Sarcopenia: European consensus on definition and diagnosis: Report of the European Working Group on Sarcopenia in Older People. Age Ageing 2010;39:412-423.

2. Prado CM, Lieffers JR, McCargar LJ, Reiman $T$, Sawyer MB, Martin L, et al. Prevalence and clinical implications of sarcopenic obesity in patients with solid tumours of the respiratory and gastrointestinal tracts: a population-based study. Lancet Oncol 2008;9:629-635.

3. van Vledder MG, Levolger $\mathrm{S}$, Ayez $\mathrm{N}$ Verhoef C, Tran TC, ljzermans JN. Body composition and outcome in patients undergoing resection of colorectal liver metastases. Br J Surg 2012;99:550-557.

4. Peng $P$, Hyder O, Firoozmand A, Kneuertz P, Schulick RD, Huang D, et al. Impact of sarcopenia on outcomes following resection of pancreatic adenocarcinoma. J Gastrointest Surg 2012;16:1478-1486.

5. Meza-Junco J, Montano-Loza AJ, Baracos VE, Prado CM, Bain VG, Beaumont C, et al. Sarcopenia as a prognostic index of nutritional status in concurrent cirrhosis and hepatocellular carcinoma. J Clin Gastroenterol 2013;47:861-870.
6. Voron $\mathrm{T}$, Tselikas L, Pietrasz D, Pigneur F, Laurent A, Compagnon P, et al. Sarcopenia impacts on short- and long-term results of hepatectomy for hepatocellular carcinoma. Ann Surg 2015;261:1173-1183.

7. Reisinger $\mathrm{KW}$, van Vugt $\mathrm{JL}$, Tegels JJ, Snijders C, Hulsewé KW, Hoofwijk AG, et al. Functional compromise reflected by sarcopenia, frailty, and nutritional depletion predicts adverse postoperative outcome after colorectal cancer surgery. Ann Surg 2015;261:345-352.

8. Kaido $T$, Ogawa K, Fujimoto $Y$, Ogura $Y$, Hata K, Ito T, et al. Impact of sarcopenia on survival in patients undergoing living 
donor liver transplantation. Am J Transplant 2013;13:1549-1556.

9. Hamaguchi $\mathrm{Y}$, Kaido $\mathrm{T}$, Okumura $\mathrm{S}$, Fujimoto $Y$, Ogawa K, Mori A, et al. Impact of quality as well as quantity of skeletal muscle on outcomes after liver transplantation. Liver Transpl Liver Transpl 2014;20:1413-1419.

10. Fujiwara $\mathrm{N}$, Nakagawa $\mathrm{H}$, Kudo $\mathrm{Y}$, Tateishi R, Taguri M, Watadani T, et al. Sarcopenia, intramuscular fat deposition, and visceral adiposity independently predict the outcomes of hepatocellular carcinoma. J Hepatol 2015;63:131-140.

11. Grignol VP, Smith AD, Shlapak D, Zhang $X$, Del Campo SM, Carson WE. Increased visceral to subcutaneous fat ratio is associated with decreased overall survival in patients with metastatic melanoma receiving anti-angiogenic therapy. Surg Oncol 2015;24:353-358.

12. Okamura $A$, Watanabe $M$, Mine $S$, Nishida $\mathrm{K}$, Imamura $\mathrm{Y}$, Kurogochi $\mathrm{T}$, et al. Clinical impact of abdominal fat distribution on prognosis after esophagectomy for esophageal squamous cell carcinoma. Ann Surg Oncol 2016;23:1387-1394.

13. Hamaguchi $Y$, Kaido $T$, Okumura $S$, Kobayashi A, Hammad A, Tamai Y, et al. Proposal for new diagnostic criteria for low skeletal muscle mass based on computed tomography imaging in Asian adults. Nutrition 2016;32:1200-1205.

14. Nishikawa $H$, Shiraki $M$, Hiramatsu $A$, Moriya K, Hino K, Nishiguchi S. Japan Society of Hepatology guidelines for sarcopenia in liver disease (1st edition): recommendation from the working group for creation of sarcopenia assessment criteria. Hepatol Res 2016;46:951-963.

15. Shen W, Punyanitya M, Wang Z, Gallagher $\mathrm{D}$, St-Onge MP, Albu J, et al. Total body skeletal muscle and adipose tissue volumes: estimation from a single abdominal cross-sectional image. J Appl Physiol 2004;97:2333-2338.

16. Ito $T$, Kiuchi $T$, Egawa $H$, Kaihara $S$, Oike $F$, Ogura $Y$, et al. Surgery-related morbidity in living donors of right-lobe liver graft: lessons from the first 200 cases. Transplantation 2003;76:158-163.

17. Morioka $D$, Egawa $H$, Kasahara $M$, Ito $T$, Haga $H$, Takada $Y$, et al. Outcomes of adult-to-adult living donor liver transplantation: a single institution's experience with 335 consecutive cases. Ann Surg 2007:245:315-325.
18. Mitsiopoulos N, Baumgartner RN, Heymsfield SB, Lyons W, Gallagher D, Ross R. Cadaver validation of skeletal muscle measurement by magnetic resonance imaging and computerized tomography. J Appl Physiol 1998;85:115-112.

19. Vehmas T, Kairemo KJ, Taavitsainen MJ. Measuring visceral adipose tissue content from contrast enhanced computed tomography. Int J Obes Relat Metab Disord 1996;20:570-573.

20. Dutkowski $P$, Linecker $M$, DeOliveira $M L$, Müllhaupt B, Clavien PA. Challenges to liver transplantation and strategies to improve outcomes. Gastroenterology 2015;148:307-323.

21. Agopian VG, Petrowsky H, Kaldas FM Zarrinpar A, Farmer DG, Yersiz $\mathrm{H}$, et al. The evolution of liver transplantation during 3 decades: analysis of 5347 consecutive liver transplants at a single center. Ann Surg 2013;258:409-421.

22. Kim WR, Lake JR, Smith JM, Skeans MA Schladt DP, Edwards EB, et al. OPTN/SRTR 2015 annual data report: liver. Am J Transplant 2017;17:174-251.

23. Toso C, Mazzaferro V, Bruix J, Freeman R, Mentha G, Majno P. Toward a better liver graft allocation that accounts for candidates with and without hepatocellular carcinoma. Am J Transplant 2014;14: 2221-2227.

24. Wiesner R, Edwards E, Freeman R, Harper A, Kim R, Kamath P, et al. Model for Endstage Liver Disease (MELD) and allocation of donor livers. Gastroenterology 2003;124:91-96.

25. Kamath PS, Kim WR. Advanced Liver Disease Study Group. The Model for Endstage Liver Disease (MELD). Hepatology 2007;45:797-805.

26. Selzner M, Kashfi A, Cattral MS, Selzner N, McGilvray ID, Greig PD, et al. Live donor liver transplantation in high MELD score recipients. Ann Surg 2010;251:153-157.

27. Weismüller TJ, Fikatas $P$, Schmidt J, Barreiros AP, Otto G, Beckebaum S, et al. Multicentric evaluation of Model for Endstage Liver Disease -based allocation and survival after liver transplantation in Germany-limitations of the 'sickest first'concept. Transpl Int 2011;24:91-99.

28. Yoshizumi T, Shirabe K, Nakagawara H, Ikegami T, Harimoto $\mathrm{N}$, Toshima $\mathrm{T}$, et al. Skeletal muscle area correlates with body surface area in healthy adults. Hepatol Res 2014;44:313-318.
29. Lexell J, Taylor CC, Sjöström M. What is the cause of the ageing atrophy? Total number, size and proportion of different fiber types studied in whole vastus lateralis muscle from 15- to 83-year-old men. J Neurol Sci 1988;84:275-294.

30. Frontera $W R$, Hughes VA, Fielding RA, Fiatarone MA, Evans WJ, Roubenoff R. Aging of skeletal muscle: a 12-yr longitudinal study. J Appl Physiol 2000;88: 1321-1326.

31. Tilg H, Moschen AR. Adipocytokines: mediators linking adipose tissue, inflammation and immunity. Nat Rev Immunol 2006; 6:772-783.

32. Lutz CT, Quinn LS. Sarcopenia, obesity, and natural killer cell immune senescence in aging: altered cytokine levels as a common mechanism. Aging (Albany NY) 2012; 4:535-546.

33. Milner JJ, Beck MA. The impact of obesity on the immune response to infection. Proc Nutr Soc 2012;71:298-306.

34. Hamaguchi $Y$, Kaido $T$, Okumura $S$, Ito $T$, Fujimoto $Y$, Ogawa K, et al. Preoperative intramuscular adipose tissue content is a novel prognostic predictor after hepatectomy for hepatocellular carcinoma. J Hepatobiliary Pancreat Sci 2015;22: 475-485.

35. Okumura $S$, Kaido $T$, Hamaguchi $Y$, Fujimoto $Y$, Masui $T$, Mizumoto $M$, et al. Impact of preoperative quality as well as quantity of skeletal muscle on survival after resection of pancreatic cancer. Surgery 2015;157:1088-1098.

36. Okumura $S$, Kaido $T$, Hamaguchi $Y$, Fujimoto $Y$, Kobayashi $A$, lida $T$, et al. Impact of the preoperative quantity and quality of skeletal muscle on outcomes after resection of extrahepatic biliary malignancies. Surgery 2016;159: 821-833.

37. Baumgartner RN, Koehler KM, Gallagher D, et al. Epidemiology of sarcopenia among the elderly in New Mexico. Am J Epidemiol 1998;147:755-763.

38. Chen LK, Liu LK, Woo J, Assantachai P, Auyeung TW, Bahyah KS, et al. Sarcopenia in Asia: consensus report of the Asian Working Group for Sarcopenia. J Am Med Dir Assoc 2014;15:95-101.

39. von Haehling S, Morley JE, Coats AJS, Anker SD. Ethical guidelines for publishing in the Journal of Cachexia, Sarcopenia and Muscle: update 2015. J Cachexia Sarcopenia Muscle 2015;6:315-316. 